\title{
Adult familial cryptogenic fibrosing alveolitis in the United Kingdom
}

\author{
Richard P Marshall, Anna Puddicombe, William O C Cookson, Geoffrey J Laurent
}

\begin{abstract}
Background-Familial cases of cryptogenic fibrosing alveolitis (CFA) have previously been reported; however, the prevalence and genetic background of this disorder are not known. The clinical and epidemiological findings of 25 families identified within the UK are reported.

Methods-Adult pulmonary physicians in the UK were asked to identify all families under their care in which two or more individuals had been diagnosed with fibrosing alveolitis of unknown cause. A detailed structured questionnaire was sent to each proband to delineate possible environmental/occupational exposures and to obtain complete pedigree data. Physicians were also asked to provide clinical and diagnostic information.
\end{abstract}

Results-Twenty five families were identified comprising 67 cases. Suitable data for analysis were available for 21 families (57 cases). The male:female ratio was $1.75: 1$ $(p<0.05)$. A high resolution computed tomographic (HRCT) scan was performed in $93 \%$ and a diagnosis of CFA confirmed on biopsy specimens in $32 \%$. The mean age at diagnosis was 55.5 (2.5) years. Fifty percent of cases were ever smokers and $18 \%$ had been diagnosed as asthmatic. Exposure to known fibrogenic agents was recorded by $36 \%$ of patients. Clinical signs/ symptoms and histological findings were indistinguishable from non-familial cases. Conclusions-This study represents the largest cohort of familial CFA cases reported to date and confirms a prevalence of 1.34 cases per $10^{6}$ in the UK population. Although rare, such cases represent an important subgroup in which a genetic susceptibility to pulmonary fibrosis is particularly evident. Familial patients are younger at diagnosis but otherwise indistinguishable from nonfamilial cases. The mode of inheritance is as yet unclear but a number of genetic loci are likely to be involved and are the subject of ongoing studies.

(Thorax 2000;55:143-146)

Keywords: genetics; cryptogenic fibrosing alveolitis

Evidence for a genetic influence in the development of interstitial pulmonary fibrosis derives from a number of observations. ${ }^{1}$ There is marked variation in response to profibrotic agents such as asbestos observed in humans, despite similar levels of exposure. ${ }^{2}$ Certain animal strains also have a susceptibility to fibrotic agents which suggests an inheritable predisposition. ${ }^{3}$ In addition, a number of rare familial forms are well documented in the literature including the Hermansky-Pudlak syndrome (characterised by oculocutaneous tyrosinase-positive albinism, platelet dysfunction and ceroid-like inclusions in the reticuloendothelial system), ${ }^{4}$ familial hypercalcaemic hypocalciuria, ${ }^{5}$ and familial cryptogenic fibrosing alveolitis (CFA). Segregation of the disease within these families suggests a major gene determinant $^{6}$; however, a simple pattern of Mendelian inheritance is not seen in most patients with CFA and a multifactorial aetiology is postulated.

No systematic study to identify familial cases has been performed to date and the true prevalence of familial CFA is unknown. Approximately 43 families have been reported in the literature worldwide, ${ }^{1}$ the largest series including just three families. ${ }^{6}$ It can be difficult to differentiate true inheritance of a disease from the mere clustering of cases within a family exposed to a common environmental factor. However, cases reported in twins ${ }^{89}$ and family members separated from an early age ${ }^{9}$ strongly suggest the existence of a genetic influence. Such cases appear histologically indistinguishable from the non-familial forms, suggesting shared pathogenic pathways. Familial syndromes are of particular interest as they are amenable to study with powerful genetic techniques including genome-wide linkage analysis and positional cloning.

In this study we have identified 25 families in the UK adult population for which clinical data were available for 21. This group had similar clinical, histological, and demographic features to non-familial cases and should be amenable to future molecular genetic analysis.

\section{Methods}

Adult pulmonary physicians (identified via the British Thoracic Society (BTS) register) were contacted by post and asked to report families in which two or more members were diagnosed with CFA. Physicians responding positively were then asked to provide further details including clinical signs of CFA, symptoms reported by the patient, and the results of diagnostic tests. In particular, details of pulmonary function testing, radiological investigation, and histological reports were obtained. The diagnosis of CFA was accepted if clinical features, bi-basal crepitations, restrictive defect on pulmonary function testing, and a lack of identifiable occupational exposure were present in combination with either compatible high resolution computed tomographic (HRCT) scans or histological appearances. 
Table 1 Demographic and clinical details of familial CFA cases

\begin{tabular}{ll}
\hline Mean (SE) age & $58(2.5)$ years \\
Mean (SE) age at diagnosis & $55(2.5)$ years \\
Male:female ratio & $1.75: 1^{\star}$ \\
Percentage ever smokers & $52 \%$ \\
Median pack years & 28.3 (range 2-75) \\
Symptoms and signs & \\
$\quad$ Breathlessness & $94 \%$ \\
Cough & $86 \%$ \\
Chest pain & $18 \%$ \\
Clubbing & $53 \%$ \\
Basal crepitations & $88 \%$ \\
${ }^{\star} \mathrm{p}<0.05\left(\chi^{2}\right.$ test). &
\end{tabular}

Patients were contacted and asked to complete a structured questionnaire (modified from that used by Scott $e t a l^{10}$ ) aimed at identifying potential environmental/occupational exposures that might modify disease onset or progression. The questionnaire included a detailed description of the geographical location and time lived in one of eight main areas of the UK, exposure to fibrogenic dusts, radiation and drugs, and a full smoking history. Further information was also requested on all additional family members representing up to four generations. This included a description of any diagnosed lung disease and demographic details to allow a family pedigree to be constructed.

\section{Results}

Eighty seven percent of pulmonary physicians responded identifying 30 possible families. Five families were excluded. In two families a condition associated with lung fibrosis was identified (rheumatological disease) and in three families a review of diagnostic tests suggested an alternative diagnosis (two with sarcoidosis and one with hypersensitivity pneumonitis). In nine of the remaining 25 families the index case died during the study but full diagnostic and limited exposure data were available for all but three of these, including four in whom a necroscopic examination was available. One patient did not respond to our request for further information and thus the final analysis was performed on data from 21 families (57 cases). All diagnoses made in relatives of index cases were made before this study.

The ratio of men to women was 1.75:1 $\left(\mathrm{p}<0.05, \chi^{2}\right.$ test). The mean (SE) age of patients at the time of the study was 58 (2.5) years and the mean age at first presentation to a respiratory physician was 55 (2.5) years (table 1). Patients were asked to record all
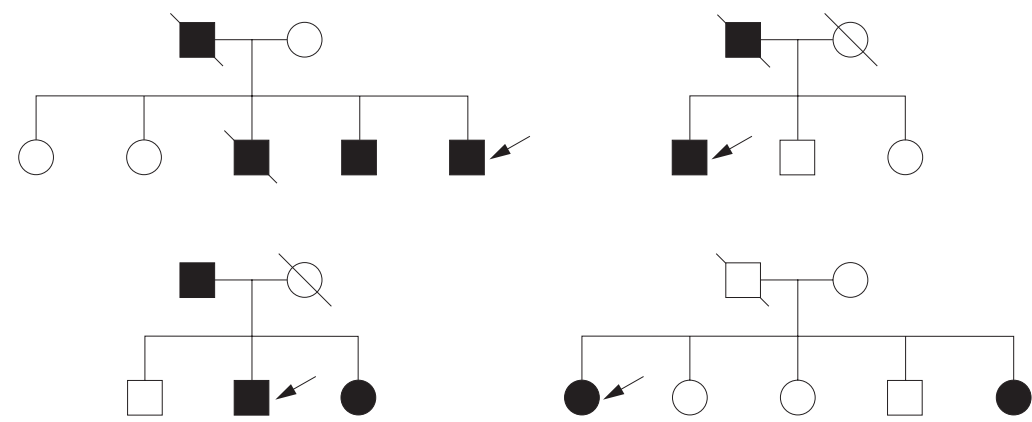

Figure 1 Representative pedigrees from subjects in four families with cryptogenic fibrosing alveolitis identified by our study. Arrows indicate index cases. places they had lived and from this a percentage time lived in one of eight regions of the UK was calculated. No cases were reported from Northern Ireland, Scotland or Wales; $76 \%$ of the total time lived by all cases was spent in the North/North West, Yorkshire and Humberside or the Midlands (East and West). The South East and South West accounted for the remaining $24 \%$.

In all cases either an HRCT scan report (93\%) and/or a lung biopsy specimen (32\%) was available. All were consistent with the diagnosis of CFA. A spectrum of HRCT patterns was reported ranging from subpleural ground glass shadowing to widespread honeycombing. Histological descriptions included varying degrees of extensive interstitial and alveolar destruction with dense matrix deposition and inflammatory cell infiltrate (cases in which granulomas were seen had previously been excluded). No attempt at subclassification into usual interstitial pneumonia, desquamative interstitial pneumonia, or non-specific interstitial pneumonia was recorded in any of the reports.

Bronchoalveolar lavage had been performed in $30 \%$ of patients. All reported a typical inflammatory cell profile with increased numbers of macrophages and an increase in the proportion of neutrophils. An increase in eosinophil count was also reported in two cases. The results of lung function testing were all consistent with the diagnosis of CFA. A reduction in forced vital capacity was the most common abnormality reported in $78 \%$. Gas transfer coefficient (KCO) was reduced in $61 \%$ of patients.

Shortness of breath (94\%) and cough (86\%) were the most common symptoms (table 1). Finger clubbing was present in $53 \%$ of patients and basal crepitations, the most common clinical sign, was present in $88 \%$. The lifetime incidence of asthma was $18 \%$. No patients reported additional atopic symptoms such as eczema or hay fever. Patients were questioned in detail regarding possible exposure to fibrogenic factors. An exposure was reported by $36 \%$ of patients. In no families did all affected members report the same exposure. A wide range of agents was implicated including coal dust, wood dust, metal dust and adhesives; the exposure was only occupational in two cases and in most the exposure was in the home. In one case occupational exposure was to asbestos but an open lung biopsy specimen showed typical features of CFA and no asbestos bodies were seen. In the second case the exposure was to wood dust. There is evidence to suggest that this may be a fibrogenic agent but it has yet to be confirmed ${ }^{10}$ and both patients were included in the study. Thirty one percent of patients had kept a pet for at least one year prior to the diagnosis of CFA. Half of the cases were ever smokers, $46 \%$ of whom were men. Median pack years smoked was 28.3 (range 2-75).

Family pedigrees were constructed (see fig 1 for representative examples). The smallest family unit included data from two siblings and both natural parents. Using the diagnosis of CFA as a marker, preliminary analysis suggests 
either an autosomal recessive mode of inheritance in those families or a dominant mode with reduced penetrance.

\section{Discussion}

This study represents the largest group of familial CFA cases published to date and confirms the rarity of the disorder. We estimate that familial cases account for $0.5-2.2 \%$ of all patients with CFA, with a prevalence of 1.34 cases per $10^{6}$ population in the UK. This is consistent with previous figures ${ }^{1}$ but may be an underestimate due to the incomplete response to our initial survey. Similarly, three cases were not included in the analysis due to a lack of accurate diagnostic information. Additional cases may also exist that have either been given alternative diagnoses or have not presented to a respiratory physician (although the number of such cases is likely to be small).

A reliance on routine clinical data for establishing the diagnosis of CFA is prone to error; however, the extent of investigation observed in this cohort is very similar to that described for the CFA population as a whole. It was not possible to differentiate between histological subtypes in CFA and thus diagnostic heterogeneity cannot be excluded.

Most of the clinical and demographic features of our cases were very similar to those reported in non-familial groups. The condition was more prevalent in men than in women, a finding similar to that in a recent BTS study. ${ }^{11}$ In contrast, a recent review highlighted studies in which the male to female ratio was close to unity. ${ }^{1}$ The reason for this disparity is not clear but could be due to chance alone or might reflect differences between the UK population and cases from the 10 countries represented by previous studies.

In this study two thirds of patients had lived in the North or Midlands regions of the UK. This distribution is somewhat different from national figures compiled by the Nottingham Study Group. ${ }^{12}$ Whether this is a genuine observation or the result of a larger at risk population from conurbated areas or differences in the identification or reporting of cases is not clear. No consistent association with environmental or occupational exposures emerged from our data.

The age of onset appears to be lower in the familial group (55 years versus 67.4 years and 69.8 years in the BTS and Trent studies, respectively). ${ }^{11}{ }^{12}$ This is similar to previous reports and could be the result of earlier diagnosis or screening in non-affected family members. However, screening was not used in these families and, given the progressive nature of CFA in the majority of patients, it is unlikely to account for all of the difference. Alternatively, the younger age may reflect a more rapid progression to clinically overt disease in the familial patients. Cases occurring in infancy, in which there is a rapid onset and progression of interstitial pulmonary fibrosis that is more responsive to immunosuppressive treatment, have also been published. ${ }^{13}$ It is likely that cases in infancy represent a distinct disease entity with a different pathogenic mechanism from that of adult onset CFA, more akin to acute interstitial pneumonia or Hamman-Rich syndrome. Alternatively, they may share a common aetiology and pathogenesis with adult CFA but possess differing clinical features, possibly the result of differences in the proliferative rate of cells in the developing lung.

A number of authors have previously proposed an autosomal dominant model with a variable penetrance but, in our cohort, an autosomal recessive pattern cannot be excluded and will require testing in the presence of further genetic data.

Do these cases support the existence of a genetic predisposition to CFA? Our current understanding of the pathogenesis of pulmonary fibrosis suggests a highly complex process involving the interaction of multiple inflammatory and repair mechanisms. Current hypotheses propose a multifactorial aetiology that implies the existence of a "fibrotic" genotype. This is likely to consist of a number of genetic factors predisposing an individual to either a reduced threshold for the development of fibrosis or an exaggerated response to a potentially fibrogenic insult.

A number of different genotypic backgrounds might lead to a similar profibrotic phenotype. Such genetic traits could be rare or of low penetrance within the general population if the environmental trigger is common. Conversely, the genotype may be of moderate or even high prevalence within a population, in which case an uncommon trigger or the need for a prolonged exposure time may be the limiting factor for the expression of the phenotype. The familial clustering evident in these cases therefore supports, but does not confirm, a significant genetic influence. Molecular genetic analysis of these cases is required to determine the extent of genotypic homogeneity.

Comparing findings, our familial cases were very similar to non-familial cases in terms of demographic and clinical features. We suggest that they form one end of a spectrum of CFA or possibly a subtype, representing patients who share a specific combination of profibrotic genetic variations or in whom these genes are more penetrant. The need for an environmental trigger is suggested by the absence of overt fibrosis in other organs (although the restriction to pulmonary disease could also be the consequence of variation in genes with tissue specific functions). In addition, the disease is manifest relatively late in life and progresses quickly in the majority of patients, arguing against a slowly progressive disorder over a longer period of time.

Many potential candidate genes exist ${ }^{14}$ including those influencing the immunological response to injury and those modulating collagen metabolism in the lung. Previous genetic studies in pulmonary fibrosis have centred on known genetic loci with a high degree of polymorphism, and those involved in the inflammatory response. The human leucocyte antigen (HLA) system, residing on chromosome 6 , is by far the most studied in this respect. The strongest correlation described is that between HLA DR3/DRw52a and the susceptibility of 
patients with systemic sclerosis to develop lung fibrosis. ${ }^{15}$ Other studies have involved only small numbers of patients, producing inconclusive and often conflicting results. ${ }^{1}$

Identifying the genes conferring a susceptibility to lung fibrosis in familial cases could improve our understanding of the pathogenic mechanisms involved in both these and nonfamilial forms. They might also aid the identification of susceptible individuals. Importantly, given the apparent similarity in the pathological features of many fibrotic lung disorders, such studies might also help to focus research towards key mediators/factors and accelerate the development of new therapeutic agents.

Our thanks to all the physicians and patients who contributed to this study and to Dr I Johnston and Professor J Britton for access to the occupational questionnaire.

This work was supported by the Wellcome Trust.

1 Marshall RP, McAnulty RJ, Laurent GJ. The pathogenesis of pulmonary fibrosis: is there a fibrotic gene? Int $\mathcal{F}$ Biochem Cell Biol 1997;29:107-20.

2 Tisdale JE, Follin SL, Ordelova A, et al. Risk factors for the development of specific noncardiovascular adverse effects associated with amiodarone. F Clin Pharmacol 1995;35: 351-6.
3 Schrier DJ, Kunkel RG, Phan SH. The role of strain variation in murine bleomycin-induced pulmonary fibrosis. $\mathrm{Am}$ Rev Respir Dis 1993;127:63-6.

4 Hermasnky F, Pudlak P. Albinism associated with hemorrhagic diathesis and unusual pigmented reticular cells in the bone marrow. Report of two cases with histochemical studies. Blood 1959;14:162.

5 Auwrex J, Demedts M, Bouillon R, et al. Coexistence of hypocalcuric hypercalcaemia and interstitial lung disease in a family: a cross-sectional study. Eur 7 Clin Invest 1985;15: $6-14$.

6 Bitterman PB, Rennard SI, et al. Familial idopathic pulmonary fibrosis. N Engl f Med 1986;314:1343-7.

7 Donohue WL, Laski B, Uchida I, et al. Familial pulmonary dysplasia and its relationship to the Hamman-Rich syndrome. Paediatrics 1959;24:786.

8 Peabody JW, Peabody JW Jr, Hayes EW, et al. Idiopathic pulmonary fibrosis: its occurrence in identical twin sisters. Dis Chest 1950;18:330.

9 Swaye P, Ordstrand HS, McCormack LJ, et al. Familial Hamman-Rich syndrome. Chest 1969;55:7-12.

10 Hubbard R, Lewis S, Richards K, et al. Occupational exposure to metal or wood dust and aetiology of cryptogenic fibrosing alveolitis. Lancet 1996;347:284-9.

11 Johnston IDA, Prescott RJ, Chalmers JC, et al. British Thoracic Society study of cryptogenic fibrosing alveolitis: current presentations and management. Thorax 1997;52 38-44.

12 Johnston I, Britton J, Kinnear W, et al. Rising mortality from cryptogenic fibrosing alveolitis. BMF 1990;301:1017-21.

$13 \mathrm{Tal}$ A, Maor E, Bar-Ziv J, et al. Fatal desquamative interstitia pneumonia in three infant siblings. F Pediatr 1984;104:873.

14 Harrison NK, Laurent GJ. Mechanisms for the pathogenesis of pulmonary fibrosis. In: Barnes PJ, ed Respiratory esis of pulmonary fibrosis. In: Barnes PJ, ed. Respiratory

15 Briggs DC, Vaughan RW, Welsh KI, et al. Immunogenetic prediction of pulmonary fibrosis in systemic sclerosis. Lancet 1991;338:661-2 